\title{
Consommations et fonctionnalités des lipides : nouveaux horizons (d'après la Journée GLN du 17 novembre 2015)
}

Le Groupe Lipides et Nutrition (GLN) est une association qui réunit chercheurs du secteur public et experts du secteur privé travaillant dans le domaine des lipides. L'objectif du GLN est de contribuer aux progrès des connaissances sur la valeur nutritionnelle des corps gras alimentaires et leurs conditions d'utilisation par l'organisme humain. Le GLN contribue ainsi tous les ans au financement de quelques projets de recherche clinique ou fondamentale sur la thématique générale Lipides et Nutrition.

Le GLN constitue un lieu de rencontres et d'échanges, notamment au travers de ses « Journées GLN », manifestations scientifiques annuelles réunissant académiques et industriels autour d'une thématique relative aux lipides et à la nutrition humaine.

En 2015, le GLN a choisi de présenter les nouveautés en matière de consommations et fonctionnalités des lipides. Par exemple la récente mise à disposition des données INCA 2 par l'ANSES a permis de calculer les apports détaillés en acides gras de la population française (oméga-3, oméga-6, détails des acides gras saturés, ... ), travail réalisé par Terres Univia en février 2015. Les participants ont également été informés des nouveaux procédés d'extraction permettant de diminuer la perte en micronutriments des huiles, des conséquences fonctionnelles des modifications des protéines par les lipides, ou encore des récentes données relatives au fonctionnement du microbiote ou au sensing des lipides.

Les lecteurs d'OCL trouveront dans ce numéro les articles de l'ensemble des intervenants de la journée GLN 2015 :

- Nouveaux procédés d'extraction et teneur en micronutriments des huiles. Florent Joffre, Jennifer Régis (ITERG - Pessac), Frédéric Fine (Terres Inovia - Pessac),

- Données récentes sur les consommations en acides gras des français. Céline Le Guillou (Terres Univia - Paris), et collaborateurs,

- Conséquences fonctionnelles des modifications covalentes des protéines par les lipides Vincent Rioux (Agrocampus Ouest, INRA USC 1378 - Rennes),

- Microbiote intestinal et obésité : impact des lipides bioactifs issus du système endocannabinoïde. Patrice Cani (Université catholique de Louvain),

- Emulsion lipidiques alimentaires et endotoxémie. Marie-Caroline Michalski, et collaborateurs (Inra UMR 1397/Inserm UMR 1060 - Villeurbanne),

- Rôle des lipides dans le développement des préférences alimentaires des enfants. Sophie Nicklaus (UMR1324 INRA Centre des Sciences du Goût et de l'Alimentation - Université de Bourgogne, Dijon),

- Perception oro-sensorielle des lipides alimentaires : impact de l'obésité. Philippe Besnard (INSERM/Université de Bourgogne/AgroSup Dijon).

La prochaine journée GLN se tiendra le mardi 15 novembre 2016 à Paris, sur le thème « Biodisponibilité et ciblage tissulaire des lipides alimentaires : nouvelles stratégies pour la formulation?». 